Voix et Images

voixetimages

\title{
La modernité revue par le rythme
}

\section{Lucie Bourassa}

Volume 21, numéro 1 (61), automne 1995

Gilles Hénault

URI : https://id.erudit.org/iderudit/201222ar

DOI : https://doi.org/10.7202/201222ar

Aller au sommaire du numéro

Éditeur(s)

Université du Québec à Montréal

ISSN

0318-9201 (imprimé)

1705-933X (numérique)

Découvrir la revue

Citer cet article

Bourassa, L. (1995). La modernité revue par le rythme. Voix et Images, 21(1),

166-171. https://doi.org/10.7202/201222ar d'utilisation que vous pouvez consulter en ligne.

https://apropos.erudit.org/fr/usagers/politique-dutilisation/ 


\title{
La modernité revue par le rythme
}

\section{Lucie Bourassa, Université de Montréal}

"Il n'y a pas si longtemps encore, les jeunes écrivains québécois ne voulaient rien considérer d'autre que la modernité, en croyant volontiers qu'elle commençait avec eux ${ }^{1}$, affirme Claude Filteau. L'essayiste fait ici allusion à "la génération des années soixante-dix", dont il dit qu'elle a reconnu, depuis, sa dette envers une modernité québécoise plus ancienne. N'empêche qu'on continue souvent à penser l'après-guerre (1948, Refus global) ou encore la Révolution tranquille comme des moments de rupture, lors desquels advient véritablement la modernité au Québec. L'étude de Filteau, qui s'attache à la poésie de la première moitié de notre siècle, contribuera certes à proposer une vision plus nuancée des choses, et à donner le goût de lire ou de relire les ouvres dont elle parle. D'autant plus qu'elle tombe bien: il existe peu de travaux sur cette période qui allient une analyse serrée des textes à une réflexion globale sur leur historicité :

\begin{abstract}
Nous chercherons moins à démontrer une évolution historique de la poésie de la modernité, qui irait du Parnasse de Bussières au *constructivisme * de Paul-Marie Lapointe, avec le passage obligé par le vers libre de Loranger et de Garneau, qu'à confronter d'un chapitre à l'autre des poètes et des critiques sur des questions de rythme, de langage, de genre pour saisir l'enjeu stratégique des débats qui entourent la question de la modernité au Québec de 1895, lors de la fondation de l'École littéraire de Montréal, jusqu'à la publication du manifeste de Borduas, Refus global, en 1948. Ce plan moins conventionnel nous permettra de multiplier au besoin les approches transverses d'une cuvre. (p. 21)
\end{abstract}

C'est ainsi que, outre les réflexions sur une œuvre précise (Arthur de Bussières, Émile Nelligan, Guy Delahaye, Jean-Aubert Loranger, Jean Charbonneau, Alfred DesRochers, Hector de Saint-Denys Garneau, Rina Lasnier, Alain Grandbois, Paul-Marie Lapointe), on trouvera des commentaires portant sur des ensembles (inventions rythmiques des parnassiens et symbolistes; éthique amoureuse de la poésie féminine de Medgé Vézina, Simone Routier, Jovette Bernier, Anne Hébert) et des études de la critique de l'époque (Louis Dantin, Camille Roy, Marcel Dugas).

Le lecteur ne doit pas s'attendre à trouver une définition de la modernité, faite d'une liste de critères - formels, rhétoriques ou idéologiques - auxquels il pourrait facilement opposer, de façon nette, ce qui n'était pas encore moderne ou ce qui ne serait plus moderne, mais postmoderne ${ }^{2}$. Quelques paramètres sont indiqués, tels que, par exemple, la conception de l'homme comme "politiquement et historiquement engendré " (p. 21); un désenchantement du monde lié à l'«échec de la rationa- 
lité ", qui attaque le "principe de raison naturelle" de saint Thomas d'Aquin, ce philosophe cher au clergé enseignant (p. 14); la question de la "liberté individuelle", que "tente de théoriser la notion de "sujet" (p. 16); l'autonomie de la littérature (p. 16), etc. Se dégage plutôt, à mesure qu'on avance dans la lecture, un portrait mouvant de la modernité telle qu'elle se constitue à travers un ensemble de tensions ou conflits internès aux discours étudiés. Elle est en particulier associée à la manière dont la voix du sujet, par les modes de signification des textes, "remet en cause les conformismes de son temps et transforme les valeurs du champ littéraire lui-même ${ }^{3}$.

Filteau emprunte à plusieurs domaines théoriques - poétique, rhétorique, grammaire, histoire des idées, philosophie, sociologie des champs littéraires -, pour se constituer une méthode de lecture. Celle-ci repose sur quelques postulats stables, exposés dans l'introduction et réexpliqués au cours des analyses. L'auteur affirme d'abord que "le sens est de l'ordre du faire et non de l'être" (p. 11), puisque l'œuvre est toujours « une traduction, une interprétation d'autres discours" (p. 19), qu'elle résulte de constants "ajustements énonciatifs". Contre une vision essentialisante, qui cherche le sens de manière immanente, Filteau suppose, avec Henri Portine, que "les phénomènes de cohérence ne sont pas dans le texte mais lisibles dans le texte ${ }^{4}$ :

Il s'agit, dès lors, d'envisager le poème comme ce qui organise la lisibilité textuelle à partir des effets d'ajustement énonciatifs qui donnent d'abord au texte la forme d'un discours faisant l'objet d'une activité de co-énonciation entre l'énonciateur et le lecteur. (p. 26)

Cette perspective "discursive" privilégie le rythme, que l'auteur associe au "passage du sujet " (p. 55), "au devenir sens d'un discours et à la voix du poème comme organisation du sens en discours" (p. 11), suivant ici les hypothèses de Meschonnic. Le choix du rythme comme fil conducteur s'explique par le fait que le renouvellement du vers et de l'émotion " fut à l'origine des premières manifestations d'une poésie moderne chez nous", alors que "les nouvelles formes du lyrisme rompaient avec le régionalisme et la grandiloquence romantique. (p. 23). Établissant les liens qui s'imposent avec la "crise de vers" française, Filteau refuse pourtant de faire de cette "crise", ou plus globalement des "changes de forme", le critère déterminant de modernité. Ces innovations ne sont pas toujours aussi patentes ici que dans la poésie hexagonale. Les choisir comme critère principal d'analyse reviendrait à privilégier une perspective de langue "comme système transcendant tout discours singulier" (p. 52), et risquerait, dans un lieu comme le Québec où romantisme, Parnasse et symbolisme "coexistent jusqu'à la veille de la Deuxième Guerre mondiale ", d'amener le critique à écarter de la modernité "des cuvres modernes par leur "contenu", mais classiques ou conventionnelles par leur "forme" ( p. 20). C'est justement le dualisme forme-contenu, emblème de 
ce que Meschonnic appelle "la métaphysique du signe" (conception instrumentale du langage comme simple apparence de ce qui est ailleurs, substitut de quelque signification sise au ciel des idées), qu'essaie d'éviter une analyse du rythme.

Si ni le rythme ni la modernité ne se réduisent à la forme, ceci ne signifie pas que les questions de métrique soient escamotées dans l'étude de ces poésies où, justement, le renouvellement du vers - donc, sa relation avec les états antérieurs - est central. Les nouvelles théorisations de la métrique fournies par la linguistique (Milner et Regnault, de Cornulier, Verluyten, Volkoff) ${ }^{5}$ décrivent celle-ci comme une "fiction linguistique" (quant à l'accentuation, les liaisons, les élisions, etc.), dérivée des règles phonologiques de la langue, mais comportant une part d'arbitraire. Cette "fiction" se transforme historiquement: elle est donc entre la "langue" (idéalité d'un modèle, d'un code) et le discours, et on peut la penser en discours, en tant qu'élément critique face à tel état du langage. Les modèles de Milner, Cornulier, Verluyten, etc., ne sont pas tous convergents, se contredisent en certains points. Le lecteur peu familier de ces nouvelles approches métriques trouvera à leur sujet, dans Poétiques de la modernité, des explications éclairantes. Filteau discute, compare, puis adopte et adapte l'un ou l'autre de ces modèles selon la situation des œuvres étudiées et l'organisation de leur écriture. Cela se fait toujours en relation avec d'autres aspects du rythme: rythme accentuel du parlé, ponctuation, typographie, rimes et séries phonétiques, car "dans un poème tout participe du sens" (p. 52). L'auteur vise par là à " construire un rapport critique entre le vers (la segmentation entre autres) et le poème comme discours associé à la mobilité du sens et du rythme" (p. 258).

L'essayiste porte aussi une attention spéciale à la, grammaire du texte, dans ses relations avec l'histoire de la grammaire: "La grammaire reconsidérée dans son histoire a [...] ceci de particulier: elle constitue l'intertexte à la fois linguistique (une théorie du langage) et extra-linguistique (un choix historique quant aux relations entre la parole et la pensée) qu'il faut articuler dans le texte" (p. 57). Elle fournit un instrument d'interprétation:

[La grammaire] est liée d'une part à ce que nous pourrions appeler le "savoir générique. du texte, du fait qu'elle indique, par exemple, les rapports qui existent entre la grammaire de la phrase et l'organisation à la fois métrique et sémantique du sonnet. Elle est liée d'autre part aux procédures de lecture qui sont plus ou moins ouvertes, mais que l'institution cherche à fixer, ne seraitce qu'au simple niveau de la ponctuation, en déterminant ce qui est acceptable ou non. (p. 86)

Ainsi, dans une étude sur "Arthur de Bussières et la grammaire des parnassiens ", on apprend que certains débats entre "parnassiens" et "symbolistes" reposent essentiellement "sur des questions de normes grammaticales". (p. 86). La question de la grammaire est d'autant plus pertinente que les relations entre langue et littérature, pour les raisons politiquies que l'on 
sait, représentent un enjeu majeur dans l'institution québécoise. Filteau évoque notamment la "politique des langues qu'adoptent l'abbé [Camille] Roy et, avec lui, la Société du parler français au Canada, fondée en 1902" (p. 15), politique qui visait à "maintenir, grâce à la littérature, la position dominante du français académique incarnant le "génie de la langue" avec, mais aussi contre les parlers régionaux" (p. 16). Divers conflits se nouent entre langue académique, parlers régionaux et possibilités d'invention dans la langue de la poésie.

Ce livre reprend le projet, cher à Meschonnic, d'une lecture, par le rythme, de l'historicité des discours. Filteau interprète toutefois davantage que ne le fait l'auteur de Critique du rythme, quii s'en tient plutôt à une description de l'organisation des textes, soulignant parfois les lieux où se manifeste l'oralité du sujet par des saturations accentuelles ou des séries prosodiques particulières. Le travail d'interprétation dans ce type d'étude est délicat, car on ne peut, sous peine de retomber dans l'ornière dualiste, "traduire " directement tel phénomène accentuel, telle manière d'employer le vers. Il faut situer, lire autrement chaque fois, les diverses composantes du texte, montrer leurs interactions, ainsi que leurs relations avec d'autres discours, ce qui pourrait exiger d'innombrables analyses. L'auteur se tire bien de la difficulté en s'arrêtant, pour chaque texte étudié, à l'une ou l'autre facette du rythme, dont il arrive à montrer le rôle clé dans la signifiance de l'œuvre: rythme de la phrase chez Arthur de Bussières; rythme sous-jacent lié à un "accent secondaire" et à une esthétique musicale chez Nelligan; rythme du continu interne à la strophe chez Loranger, qui fait sa part au "parlé "; rythme des mises en page et des réécritures chez SaintDenys Garneau.

Il serait certes trop long de résumer chacun des commentaires d'œuvres, et comme ceux-ci prennent leur sens les uns par rapport aux autres, on ne donnera qu'un aperçu bien partiel de leur richesse en évoquant les conclusions de l'un ou de l'autre. Un ou deux exemples devraient suffire à montrer la nouveauté de l'éclairage proposé, qui remet parfois en cause certaines oppositions tranchées selon lesquelles on a coutume d'envisager la poésie de cette période.

S'appuyant sur ses analyses, mais aussi, sur un classement de l'Histoire des littératures française et canadienne des sœurs de Sainte-Anne, Filteau affirme que "les débats entre exotistes et régionalistes étaient de pure rhétorique, car il s'agit de toute manière de maintenir le principe de la mimésis et la forme-vers héritée de la poésie classique" (p. 288). Dans la poésie d'Alfred DesRochers, l'analyse des rythmes montre que, tout régionaliste que soit ce poète, son vers rivalise avec celui des parnassiens, et que l'emploi de mots comme "ondoiement ", "s'oblitèrent", "témoignent de cette science des vocables que DesRochers prétend maîtriser aussi bien que tout poète exotiste» (p. 195). Si l'essayiste trouve dans cette 
œuvre des marques de la diction québécoise (quelques accents sur des prétoniques et quelques $i$ désaccentués, quelques $a$ graves à la rime [p. 195-197]), il constate aussi que cette intégration du vernaculaire se fait surtout à la périphérie des textes, dans les titres, les épigraphes et les emplois lexicaux, qu'elle n'affecte guère le compte, interdit une "diction relâchée", pour finalement assujettir le parler dialectal au parler académique: les sonnets "jouent le mètre contre le parler populaire, l'écriture savante et le style contre une prononciation populairen (p. 199). Filteau explique enfin que DesRochers s'est opposé au vers libre, "parce qu'il croyait que le vers libre se construisait contre la rime, surtout parce qu'il voyait dans la rime le moyen d'établir le rapport entre l'histoire de la langue et l'histoire de la littérature" (p. 202), mais aussi, parce qu'il espérait obtenir un prix littéraire pour son recueil, et que son choix lui assurait le soutien de plusieurs personnalités influentes.

Il fallait toute la souplesse de l'approche méthodologique et théorique de l'auteur pour montrer qu'un certain "exotisme", plus conservateur que moderniste, demeure dans la poésie régionaliste de DesRochers, ou encore, qu'à l'inverse, "les haïkus de Loranger sont à lire [...] dans le contexte de son ouvre comme un exercice de plus en vue de déplacer les rapports entre poésie et prose, en supprimant la frontière entre modernisme et régionalisme, un régionalisme qu'il démystifie par l'humour, ou qu'il associe au populismen (p. 174). La souplesse méthodologique présente aussi quelques inconvénients. Si les faits de rythme ne sont pas dans le texte, mais "lisibles dans le texte", il est certain qu'il y a plusieurs manières de les lire. L'accentuation, en particulier, est un phénomène difficile à cerner, car s'ajoutent aux accents de langue divers accents d'intensité, ainsi que, dans la versification, des lieux marqués par la métrique. En plus, contrairement à ceux de l'italien, l'anglais, ou l'allemand par exemple, les accents ne sont pas fixes en français, mais liés aux groupes syntaxiques, et seront plus ou moins rapprochés selon des facteurs tels que les genres de discours ou le débit. Filteau "négocie" avec cette difficulté de diverses manières. Il n'adopte pas toujours le même système accentuel, ne note pas toujours les mêmes choses avec les mêmes signes, et ses notations ne permettent pas toujours de distinguer, par exemple, entre les accents toniques (de fin de groupe), les accents oratoires, les accents "prosodiques" (selon Meschonnic). Le choix d'un mode de notation, quitte à l'adapter au besoin avec explications, aurait peut-être rendu plus nette la comparaison entre les rythmes des divers poètes.

Filteau, toutefois, justifie toujours son choix par la situation et la sémantique du texte, expose clairement ce qu'il marque, et arrive à convaincre, même si parfois le saut d'un modèle à l'autre est plutôt acrobatique, tant ces modèles paraissent incompatibles. Ainsi, il utilise, dans le chapitre sur "La voix du poème", la "surscansion" de Meschonnic, "qui sature le rythme de tous ses effets de sens possibles" (p. 43). Au chapitre III, 
dans l'analyse du poème "Le jardin d'antan" de Nelligan, il reprend des hypothèses métriques de Volkoff sur l'homogénéité iambique ou anapestique des hémistiches de l'alexandrin, et de Verluyten sur un "rythme sousjacent secondaire" du français: ces théories permettent de lire des iambes ou des anapestes même là où la syntaxe ne crée pas d'accents aux deux ou aux trois syllabes. Or, Meschonnic, et même des métriciens tels que Cornulier et Milner, qui voient dans la métrique un système phonologique arbitraire, mais dérivé des règles de la langue, ne pourraient souscrire à ces thèses, puisque le français n'est pas une langue à accent de mot, qui seule permet une métrique de "pieds" (iambes, anapestes, etc.). Mais ce système n'est appliqué que pour lire Nelligan, et les raisons en sont fort bien exposées: d'abord, les symbolistes s'opposent à une diction du parlé dans le vers, et la diction "cadencée, chantée, artificielle" des hémistiches iambiques ou anapestiques, qui évite de longues séquences atones ainsi que des successions "heurtées" d'accents, sert la "musique avant toute chose"; ensuite, cette diction doit être comprise "comme le passage dans la musicalité du vers de rythmes étrangers, inspirés de l'anglais, c'est-à-dire d'une langue à accents de mots" (p. 110). Nelligan, comme Verlaine et Mallarmé, parlait l'anglais. Filteau en conclut que les vers monotoniques - dont les hémistiches n'avaient, sans le rythme secondaire, qu'un accent, et qui ont fourni le point de départ de l'analyse - deviennent "dans ce contexte l'enjeu d'une dynamique rythmique "transculturelle" "(p. 110).

L'intérêt majeur de ce livre est de montrer, à travers l'analyse du rythme, comment le "renouvellement du vers et de l'émotion", non seulement donne lieu à des œuvres diverses et singulières̀, mais contribue à la transformation du paysage intellectuel, philosophique et idéologique de la modernité québécoise.

1. Claude Filteau, Poétiques de la modernité, Montréal, l'Hexagone, coll. "Essais littéraires ", 1994, p. 10. Les citations extraites de l'essai seront désormais directement suivies de leur pagination.

2. Filteau évite d'ailleurs ici de prendre parti dans les discussions sur la "postmodernité " lorsqu'il écrit : "Il n'y a pas si longtemps encore, les jeunes écrivains québécois ne voulaient rien considérer d'autre que la modernité, en croyant volontiers qu'elle commençait avec eux. Et puis le temps a passé. Sommes-nous pour autant sortis de la modernité? Je ne sais" (p. 10).

3. Comme le dit le prière d'insérer.

4. Henri Portine, "Un récit dans l'argumentation", Langue française, Paris, Larousse, $\mathrm{n}^{\circ} 50$, mai 1981, p. 127; cité par Filteau p. 26.

5. Les principales références de Filteau pour l'étude de la métrique sont les suivantes: Benoît de Cornulier, Théorie du vers. Rimbaud. Verlaine. Mallarmé, Paris, Seuil, 1982; Jean-Claude Milner, "Réflexions sur le fonctionnement du vers français", Ordre et raisons de langue, Paris, Seuil, 1982; Jean-Claude Milner et François Regnault, Dire le vers, Paris, Seuil, 1987; Vladimir Volkoff, "D'Irtamène à Dieu: la métrique de l'alexandrin hugolien ", Victor Hugo 2. Linguistique de la strophe et du vers, Minard, Lettres modernes, 1988 , p. 35-62; S. Paul Verluyten, "L'analyse de l'alexandrin. Mètre ou rythme?", M. Dominicy (dir.), Le souci des apparences, Bruxelles, Éditions de l'Université de Bruxelles, 1989, p. 31-74. 